\title{
Endoscopic ultrasound-guided liver biopsy: a multicenter experience
}

Authors

Institutions
David L. Diehl' ${ }^{1}$ Amitpal S. Johal' ${ }^{1}$, Harshit S. Khara' ${ }^{1}$, Stavros N. Stavropoulos², Mohammed Al-Haddad ${ }^{3}$, Jayapal Ramesh ${ }^{4}$, Shyam Varadarajulu ${ }^{5}$, Harry Aslanian ${ }^{6}$, Stuart R. Gordon ${ }^{7}$, Frederick K. Shieh ${ }^{1}$, Jonh J. Pineda-Bonilla ${ }^{1}$, Theresa Dunkelberger ${ }^{1}$, Dibson D. Gondim ${ }^{8}$, Eric Z. Chen ${ }^{1}$

Institutions are listed at the end of article. submitted

5. December 2014 accepted after revision 24. December 2014

\section{Bibliography}

DOI http://dx.doi.org/

10.1055/s-0034-1391412

Published online: 27.2.2015

Endosc Int Open 2015; 3: E210-

\section{E215}

(c) Georg Thieme Verlag KG

Stuttgart - New York

E-ISSN 2196-9736

\section{Corresponding author \\ David L. Diehl, MD, FACP, \\ FASGE}

Director of Interventional

Endoscopy

Department of

Gastroenterology and Nutrition

Geisinger Medical Center

100 N. Academy Avenue, 21-11,

Danville, Pennsylvania 17822

United States

Fax: +1-570-271-6852

dldiehl@geisinger.edu
Background and aims: Endoscopic ultrasoundguided (EUS) liver biopsy (LB) is proposed as a newer method that offers several advantages over existing techniques for sampling liver tissue. This study evaluated the diagnostic yield of EUSLB as the primary outcome measure. In addition, the safety of the technique in a large patient cohort was assessed.

Patients and methods: Patients undergoing EUS for evaluation of elevated liver enzymes or hepatic disease were included in this prospective, nonrandomized, multicenter study. EUS-LB was performed with EUS-fine needle aspiration (FNA; 19-gauge needle). Tissue was formalin-fixed and stained with hematoxylin and eosin, and trichrome. Using a microscope micrometer, specimen length was measured and the number of complete portal triads (CPTs) were counted. The main outcome measure was to assess the diag-

\begin{tabular}{|c|c|}
\hline \multicolumn{2}{|c|}{ Abbreviations } \\
\hline СРT & complete portal triads \\
\hline CT & computed tomography \\
\hline ERCP & $\begin{array}{l}\text { endoscopic retrograde cholangiopan- } \\
\text { creatography }\end{array}$ \\
\hline EUS & endoscopic ultrasound \\
\hline FNA & fine needle aspiration \\
\hline $\mathrm{H} \& \mathrm{E}$ & hematoxylin and eosin \\
\hline $\mathrm{HCV}$ & hepatitis C virus \\
\hline INR & international normalized ratio \\
\hline LB & liver biopsy \\
\hline MRCP & $\begin{array}{l}\text { magnetic resonance cholangiopancrea- } \\
\text { tograpy }\end{array}$ \\
\hline NAFLD & nonalcoholic fatty liver disease \\
\hline NASH & nonalcoholic steatohepatitis \\
\hline
\end{tabular}

nostic yield of EUS-LB, and to monitor for any procedure-related complications.

Results: Patients (110; median age, 53 years; 62 women) underwent EUS-LB at eight centers. The indication was abnormal liver enzymes in 96 patients. LB specimens sufficient for pathological diagnosis were obtained in 108 of 110 patients (98\%). The overall tissue yield from 110 patients was a median aggregate length of $38 \mathrm{~mm}$ (range, 0-203), with median of 14 CPTs (range, $0-68$ ). There was no statistical difference in the yield between bilobar, left lobe only, or right lobe only biopsies. There was one complication (0.9\%) where self-limited bleeding occurred in a coagulopathic and thrombocytopenic patient. This complication was managed conservatively.

Conclusions: EUS-guided LB was a safe technique that yields tissue adequate for diagnosis among $98 \%$ of patients evaluated.

\section{Introduction}

Liver biopsy (LB) has historically been performed percutaneously without image guidance, also known as "blind biopsy". In the past several years, there has been more reliance on image guidance to direct the needle into the liver to limit the risk of complications [1]. The percutaneous route is typically directed by ultrasound or computed tomography (CT), and a transjugular fluoroscopyguided approach is used if the percutaneous route is not safe because of coagulopathy or ascites, or if measurement of portal pressure is required [2]. Surgical LB (either laparoscopic or open) is yet another way of obtaining liver tissue.

A newer method of obtaining a LB is by endoscopic ultrasound (EUS)-guidance [3-7]. EUS provides a high resolution image of both lobes of the liver, and a biopsy needle can be safely directed into the liver for sampling under image guidance. Doppler capability of the linear echoendoscope allows intrahepatic vessels to be avoided 

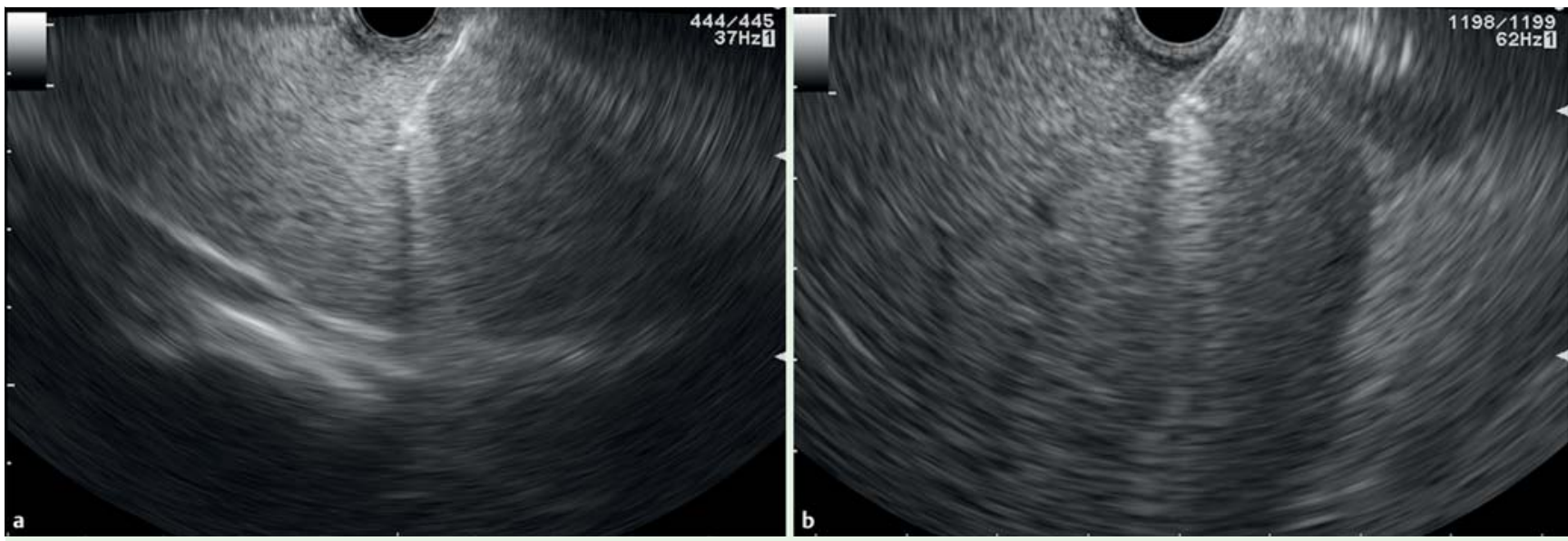

Fig. 1 EUS-LB using fine needle aspiration (19-gauge needle). a The left lobe of the liver. $\mathbf{b}$ The right lobe of the liver.

during FNA. Intervening structures such as loops of bowel, gallbladder, and the pleural space can be easily observed and avoided, further lowering the risk of complications. Another potential advantage of EUS-LB is that it easily and safely allows biopsy of both left and right lobes of the liver, potentially addressing concerns about sampling error. In addition, EUS-LB uses a 19-gauge needle, which is smaller than the 16-gauge needles that have traditionally been used for transcutaneous LB [1], and this may provide an additional measure of safety.

Many studies that have used a 19-gauge Tru-cut biopsy or FNA needle to acquire liver tissue have obtained specimens adequate for histologic diagnosis [3-7]. In the most recently reported series, a 19-gauge FNA needle was used in 22 patients and yielded adequate tissue for histological analysis in 20 (91\%) of them and there were no complications [6]. Aside from these few reports, there has not been a larger study on the effectiveness and safety of EUS-LB. This report describes the results of a multicenter experience with the yield and safety of EUS-LB.

\section{Patients and methods}

Consecutive patients who underwent EUS-LB at the participating centers were included in our prospective, non-randomized, multicenter study. Each participating center had Institutional Review Board approval to participate in this study. Inclusion criteria were patients who had undergone extensive previous serological and/ or cross sectional imaging which were non-diagnostic for their underlying presentation, leading to a decision for subsequent EUS to evaluate for elevated liver enzymes or hepatic parenchymal disease. Consent from the patient was obtained for a possible EUS-LB or endoscopic retrograde cholangiopancreatography, if needed, to be done at the time of the EUS - a decision based on the EUS findings. If EUS evaluation of the liver, hepatobiliary tree, gallbladder (if present), ampullary area, and pancreas was nondiagnostic, a decision was made to proceed with the EUS-LB. If EUS showed a biliary obstruction, then in the same session endoscopic retrograde cholangiopancreatography, was performed to treat the obstruction and EUS-LB was not performed. Exclusion criteria included malignant liver disease, thrombocytopenia (platelets, $<50,000$ ), or coagulopathy (international normalized ratio [INR] > 1.5), use of antiplatelet agents within 5 days of the procedure, inability to provide informed consent, or pregnant status. Patient demographics, procedure information, diagnostic yield, specimen adequacy, and complication rates were recorded. Pa- tients were contacted at 1-2 days and 30 days post-procedure to check for procedure-related complications.

\section{FNA technique}

EUS examination was performed in the left lateral decubitus position with a linear echoendoscope (GF-UC140P, Olympus America, Center Valley, PA, United States). All patients underwent deep sedation with propofol administered by a staff anesthesiologist and/or nurse anesthetist. Pre-procedure antibiotics were not given. The biopsy was performed using a 19-gauge EUS-FNA needle (19G Expect or 19G Expect Flexible, Boston Scientific, Marlborough, MA, United States). This was done with or without a stylet as per the endoscopist's preference. Almost all endoscopists preferred to use full suction for the needle aspiration. The left lobe of the liver was accessed with the echoendoscope in the proximal stomach, distal to the gastroesophageal junction. The right lobe of the liver was accessed with the echoendoscope positioned in the duodenal bulb and torqued counter clockwise, beyond the view of the portal vein and the gallbladder (if present). Doppler was used to identify an area of hepatic parenchyma in the expected trajectory of the needle that was free of blood vessels or bile ducts ( $\bullet$ Fig. 1 ). After initial puncture, 7-10 back-and-forth motions of the needle were made per pass using the "fanning" technique to maximize tissue sampling. This technique is well described for EUS-FNA, and involves changing the trajectory of the needle with each back-and-forth movement. One or two passes were made in the left lobe depending on the endoscopist's preference or assessment of tissue yield after the first pass. Right lobe FNA, when performed, was per endoscopist's preference, using the same technique. After completion of the EUS-LB, the patient was observed in the endoscopic recovery unit for 1-3 hours, depending on the endoscopist's preference, and discharged if no immediate complications were noted.

\section{Specimen handling}

After completion of one biopsy pass, tissue was expressed from the needle with the stylet directly into formalin without additional tissue handling. The contents of the formalin container were inspected for visible tissue cores ( $\mathbf{F i g} . \mathbf{2 a}$ ). Tissue processing was as routinely performed at the surgical pathology laboratory. The surgical pathology technician poured the contents of the formalin container into a petri dish ( $\bullet$ Fig. $\mathbf{2 b}$ ). Visible pieces of liver tissue were identified, removed with forceps ( $\bullet$ Fig. 2c), placed in lens paper $(\bullet$ Fig. $\mathbf{2 d}$ ), and submitted in a histology cas- 


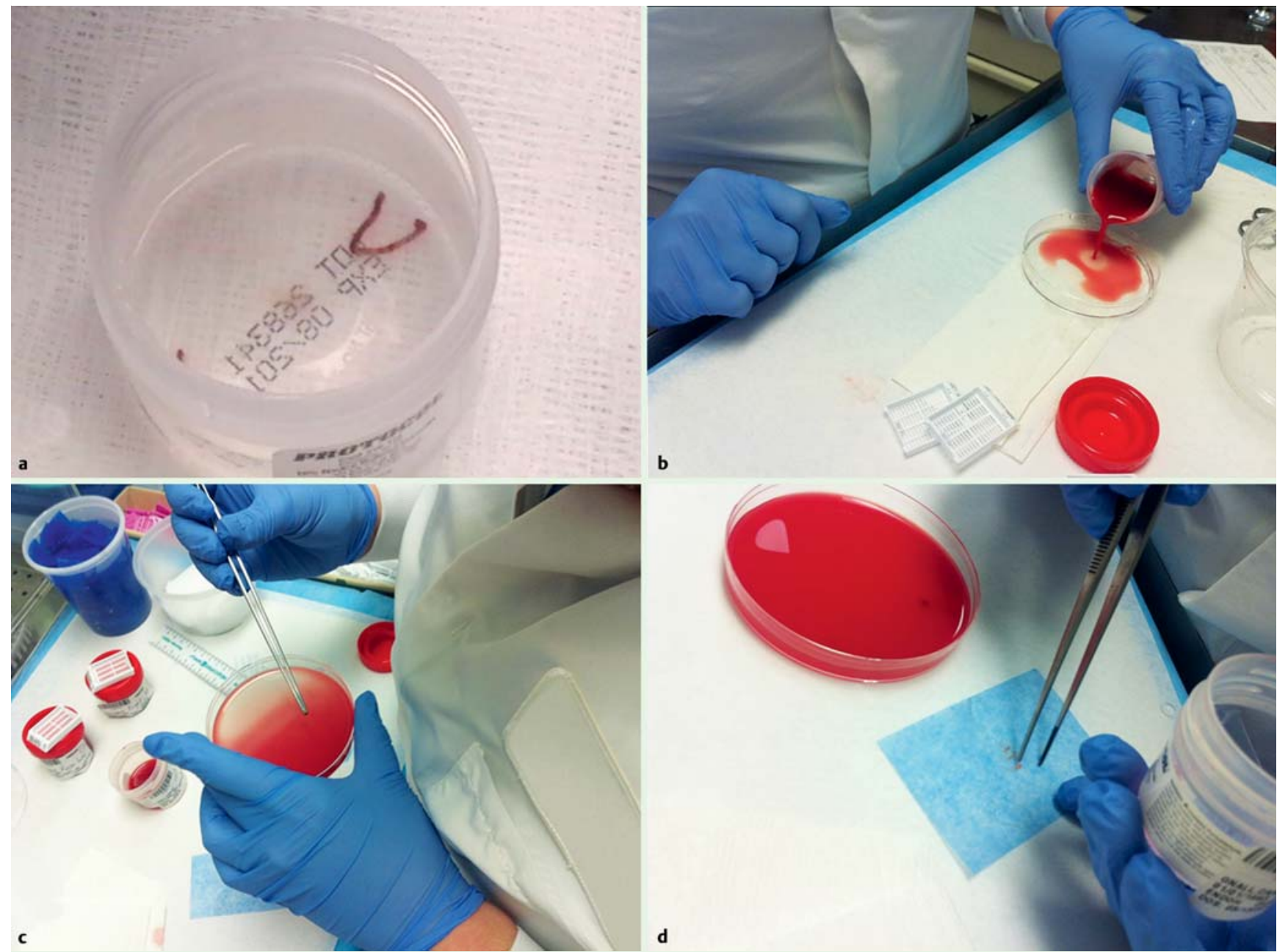

Fig. 2 Tissue was expressed into the formalin container. a Visible core. $\mathbf{b}$ More bloody specimens; the contents of the container were poured into a petri dish c The formalin-fixed pieces of liver tissue, distinguished from blood clot, were removed with forceps. $\mathbf{d}$ Next, the tissue was wrapped in lens paper and placed into a histology cassette for standard processing; the blood clot was also submitted. Usually, all material was processed in a single cassette.

sette for processing. The liver tissue was distinguished from blood clots by its color. All the remaining tissue/clot was submitted in additional cassette(s). The tissue was fixed and embedded in paraffin; $4 \mu \mathrm{m}$ sections were made with a microtome and glass slides were prepared. The slides were stained for histologic evaluation (typically, hematoxylin and eosin [H\&E], reticulin, and trichrome, with Periodic acid-Schiff-diastase and iron stain as needed in selected patients).

\section{Quantification of tissue yields}

Using a microscope micrometer, specimen lengths (both cumulative length and longest piece) were measured and number of complete portal triads (CPTs) counted. This was done primarily on trichrome-stained slides, which highlight the portal triads, although H\&E-stained slides were analyzed if trichrome was not available. In some patients, whole slide scans were made with a digitizing scanner (ScanScope CS, Aperio Technologies, Inc. Vista, California, United States) and specific slide viewing software (Aperio Image Scope Viewer, version 11.2.0.780) was used for the quantitative analysis. With this software, an internal digital measurement function allows for precise quantification of liver core lengths $(\bullet$ Fig. $\mathbf{3 a}$ ). A dedicated gastrointestinal pathologist was blinded to the clinical information and reviewed the specimens.

\section{Study definitions}

Complications of EUS-LB were defined as any deviation from the expected post-procedure clinical course, and were prospectively assessed by post-procedure phone calls at $1-2$ days and 30 days after the procedure. Complications included bleeding, perforation, pneumothorax, bile leak, or infections [8]. Sedation-related complications were also documented. A bleeding complication was defined as symptomatic blood loss requiring further investigation, transfusion, or admission to hospital. Diagnostic yield was defined as a sufficient amount of liver tissue on the slide to allow the pathologist to make a diagnostic interpretation.

\section{Statistical analysis}

The study data were collected on an Excel spreadsheet and descriptive statistics were utilized to represent continuous and categorical variables, whereby results were expressed as medians with ranges. Multiple comparisons between the aggregate tissue length and CPT yield from bilobar, left lobe only, and right lobe only biopsies were carried out using the Mann-Whitney-Wilcoxon test. A $P$-value of $<0.05$ was considered statistically significant.

\section{Main outcome measures}

Main outcomes measures were to assess the biopsy specimen for diagnostic adequacy and procedural complications. 


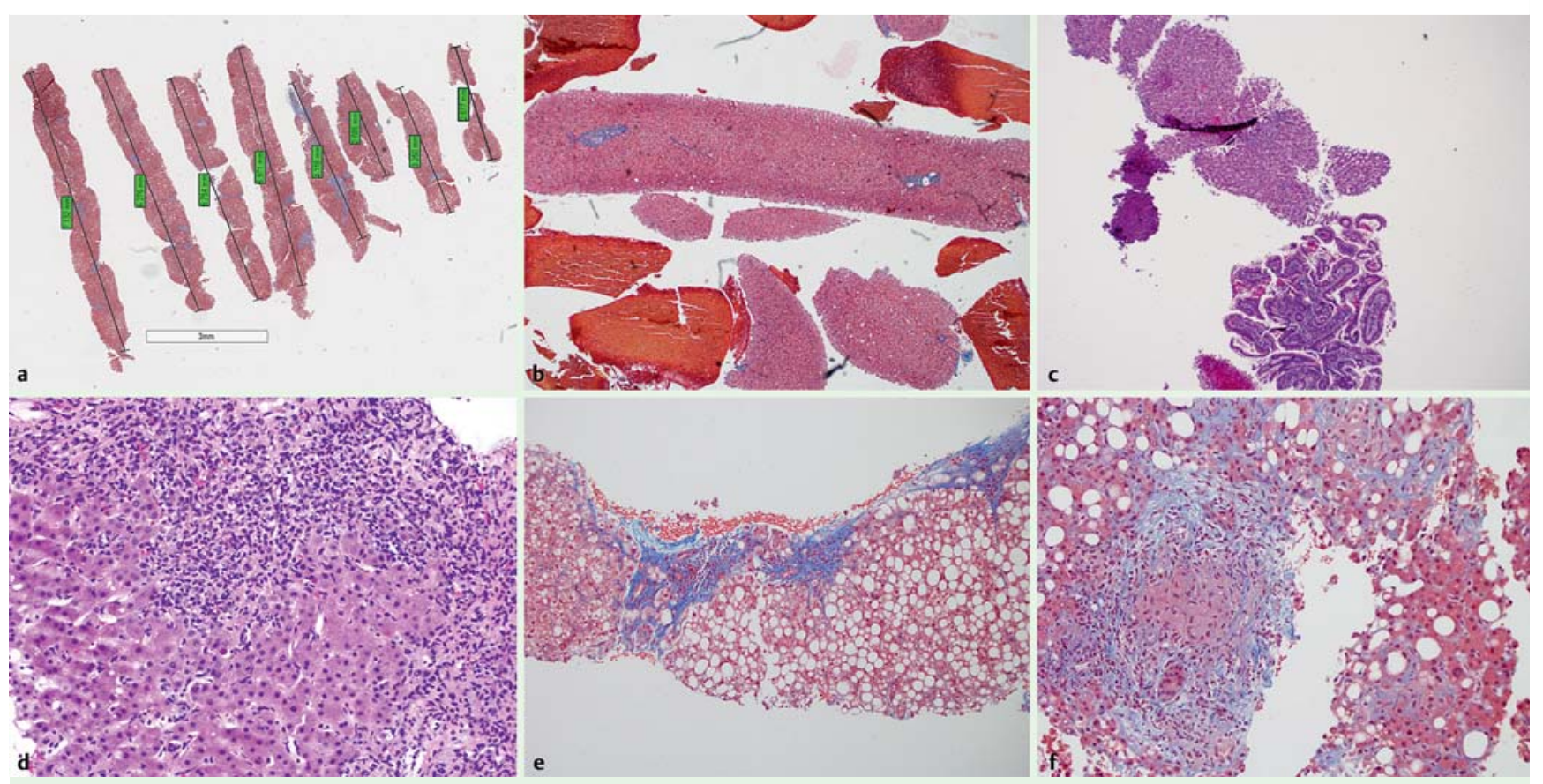

Fig. 3 Precise quantification of liver core lengths. a Digital measurements. b Representative photomicrographs showing liver tissue mixed with blood clot obtained from patients from this series. $\mathbf{c}$ Duodenal mucosa with right lobe EUS-LB; $\mathbf{d}$ Autoimmune hepatitis under high power view. e Steatohepatitis. $\mathbf{f}$ Noncaseating granulomatous hepatitis.

\section{Results}

$\nabla$

Between November 2011 and September 2013, one hundred and ten patients (62 women; median age, 53 years [range, 9-87]) underwent EUS-LB at the eight participating centers. The most common indication for performing EUS-LB was persistent unexplained elevation in serum transaminases $(n=96)$, followed by evaluation of hepatic parenchyma for suspected underlying liver disease $(n=14)$.

Biopsies of both the left and the right lobe in the same patient were performed in 68 (62\%) of 110 patients, left lobe only biopsies were done in 34 (31\%) patients, and right lobe only biopsies were done in 8 (7\%) patients. Two patients with Roux-en-Y gastric bypass had successful left lobe only biopsies because of their altered anatomy and the inability to access the right lobe. The overall yield from 110 patients was a median aggregate tissue length of $38 \mathrm{~mm}$ (range, $0-203$ ), with a median of 14 CPTs (range, 0-68). The individual yields of bilobar, left lobe only, and right lobe only biopsies are summarized in $\bullet$ Table 1 . There was no statistical difference in the aggregate tissue length (left vs. right, $P=0.44$; bilobar vs. right, $P=0.99$; bilobar vs. left, $P=0.06$ ) the number of CPTs (left vs. right, $P=0.58$; bilobar vs. right, $P=$ 0.65 ; bilobar vs. left, $P=0.12$ ) yielded by any of these access methods.

Three patients who underwent bilobar biopsies had no tissue yield from one of the lobes (two patients with no tissue from right lobe and one patient with no tissue from left lobe), but they were analyzed as bilobar biopsies because there was adequate liver tissue available for histological analysis from the other lobe. There was one patient who underwent a left lobe only biopsy where adequate tissue was not obtained for histopathological analysis, and one patient who underwent a bilobar biopsy that yielded $23 \mathrm{~mm}$ aggregate tissue but only 5 CPTs, which was inadequate for establishing a histopathological diagnosis. The remaining 108 (98\%) of 110 patients yielded specimens sufficient
Table 1 Quantified yields from endoscopic ultrasound liver biopsies.

\begin{tabular}{|c|c|c|c|}
\hline & Patients & $\begin{array}{l}\text { Median tissue length, } \\
\mathrm{mm} \text { (range) }\end{array}$ & $\begin{array}{l}\text { Median CPTs } \\
\text { (range) }\end{array}$ \\
\hline Both lobes & 68 & $40(9-148)$ & $17(65)$ \\
\hline Left only & 34 & $32(0-203)$ & $13(0-68)$ \\
\hline Right only & 8 & $49(8-113)$ & $14(6-34)$ \\
\hline
\end{tabular}

CPTs, complete portal triads.

for definitive pathological diagnosis ( $\bullet$ Table 2 ). There were only five patients where the tissue yield was less than 6 CPTs with aggregate length less than $15 \mathrm{~mm}$; the value used as a reference standard in recent studies [4-6]. However, we were able to obtain a histological diagnosis in samples from four, but not from the one of the five patients because of the complete lack of tissue on that biopsy. Representative microscopic images from several patients demonstrate the general size of the liver tissue obtained and some of the pathology encountered ( $\mathbf{F i g . 3 b}-\mathbf{f}$ ).

The overall complication rate from EUS-LB noted in our study was $1 / 110(0.9 \%)$. The single complication occurred in a coagulopathic and thrombocytopenic patient (platelets, 64,000; INR, 1.42), evaluated for abnormal transaminases. Post-procedural abdominal pain was observed, which required evaluation with CT scan that showed a pericapsular hematoma. Angiogram was subsequently performed but did not show any active bleeding, thus angioembolization was not required. This patient was eventually found to have disseminated intravascular coagulation. No other complications were reported in any patient. 
Table 2 Diagnoses obtained from the EUS-LB.

\begin{tabular}{|l|l|}
\hline Diagnosis & Patients \\
\hline Nonalcoholic fatty liver disease & 52 \\
$\quad$ Nonalcoholic steatohepatitis (NASH) & 30 \\
$\quad$ Steatosis without NASH & 22 \\
\hline $\begin{array}{l}\text { Viral hepatitis } \\
\quad \text { Chronic }\end{array}$ & 11 \\
$\quad$ Acute & 1 \\
\hline Drug induced liver injury & 9 \\
\hline Non-specific cholestasis & 8 \\
\hline Non-specific mild inflammation & 5 \\
\hline Normal liver & 3 \\
\hline Autoimmune hepatitis & 3 \\
\hline Granulomatous hepatitis & 3 \\
\hline Insufficient sample & 2 \\
\hline Acute cholangitis & 2 \\
\hline Iron overload & 2 \\
\hline Pericholangitis & 1 \\
\hline Acute alcoholic hepatitis with cirrhosis & 1 \\
\hline Cirrhosis from HCV and NASH & 4 \\
\hline Post orthotopic liver transplant, & \\
\hline no acute rejection & 1 \\
\hline
\end{tabular}

$\mathrm{NASH}$, nonalcoholic steatohepatitis; $\mathrm{HCV}$, hepatitis C virus.

\section{Discussion}

$\nabla$

There are several potential advantages of EUS-LB, which can be performed in an outpatient setting and offers the comfort of sedation and analgesia, thereby reducing pain and anxiety. Multiple sites can be sampled, which can provide a more accurate representation of liver histology. It has been recognized that sampling error can lead to diagnostic inaccuracy of a biopsy from a single site [9]. The left hepatic lobe is more easily accessed for biopsy by EUS than by CT or percutaneous ultrasound-guided needle biopsy, because of the larger surface area which can be scanned by EUS for an optimal biopsy site. Another potential advantage is its safety because the area of liver being sampled can be interrogated in "real-time" under ultrasound guidance, and intervening vessels and organs can be avoided. In addition, because the patient is sedated for the EUS procedure, the LB is less uncomfortable than a percutaneous un-sedated approach.

In a patent who is already undergoing an esophagogastroduodenoscopy or EUS for their respective indication, EUS-LB can be performed with little additional time and risk. This approach can spare the patient the additional discomfort and expense of a second dedicated LB procedure. Equipment costs for the EUS-LB include only the FNA needle, which is similar to or less expensive than the cost of needles for the transjugular or percutaneous approach. More tissue accessed with the EUS-FNA fanning technique than with the single pass percutaneous technique. However, the cost of the endoscopic procedure makes up a significant part of the expense of EUS-LB. Thus, it seems this approach is best for patients requiring $\mathrm{LB}$ who are already undergoing an upper endoscopy and/or EUS exam or who require sedation for a LB.

Although magnetic resonance cholangiopancreatography (MRCP) and EUS are comparable in their diagnostic utility for evaluation of hepatobiliary diseases, the need for use of contrast and the inability to provide a histological diagnosis are limitations of MRCP. Moreover, if MRCP is found to be negative for biliary obstruction, there would be a subsequent need for liver histology, which requires a separate procedure with added cost. Thus, the workup of all our study patients was initiated as a diag- nostic EUS and EUS-LB was performed as needed. This eliminated the need for a separate procedure for tissue acquisition and thereby reduced the cost to the patient.

Among patients who have had a Roux-en-Y gastric bypass, EUSLB can still be successfully performed with a transgastric approach via the gastric pouch to accurately target the left hepatic lobe. This was done in three of the patients in our series with good diagnostic yield. Among patients who may be living donor candidates, the specific lobe for donation can be targeted by EUSLB for pre-transplant histological evaluation.

Several of the initial reports of EUS-LB utilized a spring loaded 19-gauge core needle (Quick Core, Cook Medical, Winston-Salem, North Carolina, United States) [3-5]. In the hands of these investigators, tissue yields were satisfactory but there was a wide range of specimen adequacy $(19-100 \%)$. This device has been associated with several technical difficulties that could reflect negatively on tissue adequacy. The ability to use a regular 19gauge EUS needle in 22 patients with no complications and to obtain adequate liver specimens was first described by Stavropoulos et al [6]. They sampled only the left lobe, but did use multiple passes of the needle with suction to obtain tissue, which served as the basis of our protocol as well. This preliminary finding led to the current multicenter study to see if the results from a single center, single endoscopist study could be generalizable.

We found that the EUS-LB technique was successful and safe for obtaining adequate liver biopsy specimens. The single patient who experienced clinical bleeding that was observed in this series occurred in a patient with thrombocytopenia and ongoing coagulopathy that was later identified as disseminated intravascular coagulation. This patient may have encountered bleeding irrespective of the technique adopted to sample the liver. Although our study included five patients with cirrhosis, none of the patients had ascites, thus we cannot comment on the safety of EUS-LB in the presence of ascites. We believe that the EUS-LB technique may not be appropriate for patients with coagulopathy or thrombocytopenia and in whom there is need for portal pressure measurement with liver biopsy.

Adequacy of a LB specimen remains a qualitative assessment by the pathologist. Standards for quantitative adequacy have been established by the American Association for the Study of Liver Diseases [1] and these quantitative parameters include length of specimen and the yield of CPTs that can be identified in the specimen. Current published studies on EUS-LB have used 6 CPT's or a mean length of $15 \mathrm{~mm}$ as criteria for sample adequacy [4-6]. However these parameters are based on results of prior studies using a large-bore percutaneous needle. Thus, it is not clear how generalizable these results may be. A recent study of ten patients showed that EUS-LB using a 19-gauge FNA needle had 100\% diagnostic yield with an average of 9.2 CPTs and average core length of $14.4 \mathrm{~mm}$ [10]. However, we believe that diagnostic yield should be based on the ability of a pathologist to successfully render a diagnosis on the material provided, because a diagnosis was successfully rendered for our patients with low CPTs $(<6)$ and core lengths ( $<15 \mathrm{~mm}$ ), whereas one patient with $23 \mathrm{~mm}$ tissue length had a non-diagnostic sample.

The indications for the use of LB appear to be increasing. Recently, there has been recognition that nonalcoholic fatty liver disease (NAFLD) can lead to liver damage such as nonalcoholic steatohepatitis (NASH) and cirrhosis. NASH is an increasingly common cause of liver failure needing liver transplantation with higher liver-related mortality than simple steatosis [11]. Many hepatologists are recommending early biopsy in patients with NAFLD to 
stage the degree of hepatic fibrosis and to help with decisions regarding starting therapy. In the future, as additional therapies become available for NASH, the need for and use of LB will undoubtedly increase. The EUS-LB approach may be cost effective in patients with NAFLD who are being considered for liver biopsy and who are also in need of EUS for other non-hepatic indications.

This multicenter study confirmed that EUS-LB is a safe procedure and yields tissue adequate for histologic diagnosis. The technique can be useful for patients undergoing EUS for evaluation of elevated liver enzymes or hepatic disease. Comparative studies to evaluate tissue yields with other LB techniques and with matched subjects may be useful to further define the role of EUS in patients undergoing LB.

\section{Competing interests: None}

\section{Institutions}

${ }^{1}$ Geisinger Medical Center, Department of Gastroenterology and Nutrition, Danville, Pennsylvania, United States

${ }^{2}$ Winthrop University Hospital, Departments of Gastroenterology, Hepatology and Nutrition, Mineola, New York, United States

${ }^{3}$ Indiana University, Department of Gastroenterology and Hepatology, Indianapolis, Indiana, United States

${ }^{4}$ University of Alabama at Birmingham, Division of Gastroenterology and Hepatology, Birmingham, Alabama, United States

${ }^{5}$ Florida Hospital, Center for Interventional Endoscopy, Orlando, Florida, United States

${ }^{6}$ Yale University School of Medicine, Department of Medicine, Section of Digestive Diseases, New Haven, Connecticut, United States

${ }^{7}$ Dartmouth-Hitchcock Medical Center, Department of Gastroenterology, Lebanon, New Hampshire, United States

${ }^{8}$ Indiana University, Department of Pathology and Laboratory Medicine, Indianapolis, Indiana, United States

\section{References}

1 Rockey DC, Caldwell SH, Goodman ZD et al. AASLD Position Paper: Liver Biopsy. Hepatology 2009; 49: 1017-1044

2 Kalambokis G, Manousou P, Vibhakorn S et al. Transjugular liver biopsy - Indications, adequacy, quality of specimens, and complications - A systematic review. J Hepatol 2007; 47: 284-294

3 Mathew A. EUS-guided liver biopsy in select patients. Am J Gastroenterol 2007; 102: $2354-2355$

4 Gleeson FC, Clayton AC, Zhang $L$ et al. Adequacy of endoscopic ultrasound core needle biopsy specimen of nonmalignant hepatic parenchymal disease. Clin Gastroenterol Hepatol 2008; 6: 1437-1440

5 Dewitt J, McGreevy K, Cumming $O$ et al. Initial experience with EUSguided Tru-cut biopsy of benign liver disease. Gastrointest Endosc 2009; 69: 535-542

6 Stavropoulos SN, Im GY, Jlayer Z et al. High yield of same-session EUSguided liver biopsy by 19-gauge FNA needle in patients undergoing EUS to exclude biliary obstruction. Gastrointest Endosc 2012; 75: $310-318$

7 Nakai Y, Samarasena JB, Iwashita T et al. Autoimmune hepatitis diagnosed by endoscopic ultrasound-guided liver biopsy using a new 19gauge histology needle. Endoscopy 2012; 44: E67-E68

8 Adler DG, Jacobson BC, Davila RE et al. ASGE Guideline: complications of EUS. Gastrointest Endosc 2005; 61: 8-12

9 Maharaj B, Maharaj RJ, Leary WP et al. Sampling variability and its influence on the diagnostic yield of percutaneous needle biopsy of the liver. Lancet 1986; 1: $523-525$

10 Gor N, Salem SB, Jakate $S$ et al. Histological adequacy of EUS-guided liver biopsy when using a 19-gauge non-Tru-Cut FNA needle. Gastrointest Endosc 2014; 79: 170-172

11 Musso G, Gambino E, Cassader M et al. Meta-analysis: natural history of non-alcoholic fatty liver disease (NAFLD) and diagnostic accuracy of non-invasive tests for liver disease severity. Ann Med 2011; 43: $617-$ 649 